\title{
Evaluation of the Employment of UAVs as Fog Nodes
}

This paper was downloaded from TechRxiv (https://www.techrxiv.org).

\section{LICENSE}

CC BY-NC-SA 4.0

SUBMISSION DATE / POSTED DATE

04-02-2021 / 06-02-2021

CITATION

Cardoso da Silva, Rodrigo Augusto; da Fonseca, Nelson Luis Saldanha; Boutaba, Raouf (2021): Evaluation of the Employment of UAVs as Fog Nodes. TechRxiv. Preprint. https://doi.org/10.36227/techrxiv.13717792.v1

$\mathrm{DOI}$

10.36227/techrxiv.13717792.v1 
This work has been submitted to the IEEE for possible publication. Copyright may be transferred without notice, after which this version may no longer be accessible.

\title{
Evaluation of the Employment of UAVs as Fog Nodes
}

\author{
Rodrigo A. C. da Silva, Nelson L. S. da Fonseca, and Raouf Boutaba
}

\begin{abstract}
In fog computing, processing, network, and storage resources are placed close to the end users to assure a low latency in comparison to the latency experienced when accessing services in the cloud. One limitation of this solution, however, is that fog nodes are usually fixed, whereas demands are variable over time at all locations, resulting in underutilization of the fog resources as well as unnecessary provisioning of fog resources. One way for dealing with this problem is the employment of mobile nodes to cope with the variability in resource demand. This paper studies how unmanned aerial vehicles (UAVs) equipped with processing capabilities can be used in this perspective, and proposes a solution to the fog node location problem considering both fixed and mobile nodes. It proposes the UAV Fog Node Location (UFL) algorithm to evaluate potential replacements of fixed servers by UAVs. The proposed algorithm can be used for long term planning under the assumption of changes in the prices of UAVs. An evaluation of the problem using data generated by real mobile users shows that UAVs can improve the design of future fog networks.
\end{abstract}

\section{Index Terms}

Facility location, fog computing, unmanned aerial vehicle

\section{INTRODUCTION}

Cloud computing has facilitated the deployment of numerous online services as well as services to augment the capabilities of user devices [1]. Cloud computing relies on large data centers that host computing, network, and storage resources accessed on demand through the Internet. However, they are typically located in remote areas making a variety of applications with strict latency requirements

This research was funded by Google Latin American Research Awards 2019, the Government of Canada through the Emerging Leaders in the Americas Program (ELAP), National Council for Scientific and Technological Development (CNPq) grant number 140464/2018-2, and São Paulo Research Foundation (FAPESP) grant 15/24494-8.

Rodrigo A. C. da Silva and Nelson L. S. da Fonseca are with the Institute of Computing, State University of Campinas, Campinas, Brazil (e-mail: rodrigo@1rc.ic.unicamp.br; nfonseca@ic.unicamp.br). Raouf Boutaba is with the Cheriton School of Computer Science, University of Waterloo, Waterloo, Canada (e-mail: rboutaba@uwaterloo.ca) 
unfeaseable. One solution to alleviate this limitation is the employment of fog computing, an architecture to provide computing, storage, and networking capabilities anywhere along the continuum between the cloud and the end users [2]. Fog nodes are usually deployed as fixed nodes in different locations to support the computational needs of local users [3] and support the strict latency requirements of applications. However, nodes in a fixed infrastructure may need to be over-dimensioned to cope with variable processing demands. Unmanned aerial vehicles (UAVs) have been employed for military applications, surveillance, and traffic analysis to name a few. More recently, UAVs have been considered for integration into cellular networks to serve as base stations [4], [5], allowing providers to expand their coverage area in case of occasional demands or failure of the terrestrial infrastructure. The employment of UAVs as fog nodes is still its infancy. A few studies have considered such a use [6], [7], but they have not modeled the limited autonomy of UAVs, which restricts their usability in real infrastructures. A detailed battery model for UAVs needs to be accounted for in the evaluation of their potentiality as processing nodes.

This paper aims at assessing the advantages of employing UAVs as fog nodes for dealing with variable workload demands generated by mobile users. In this perspective, this paper studies the problem of where to locate UAVs as fog nodes (the fog node location problem) in a metropolitan area with the aim of offering cloud services at the edge. In contrast to previous approaches [8], [9], this paper considers both fixed nodes and mobile UAV nodes. Fixed nodes are always available due to continuous energy supply, but, once deployed, they cannot be easily migrated to another location. On the other hand, UAV nodes can fly between different locations to augment the processing capacity of fixed fog nodes, especially to process workload in excess of the capacity of fixed nodes. However, UAV nodes are mobile and operate on batteries which limit the length of time they can process user workload. The consideration of both fixed and mobile nodes enables the deployment of an infrastructure capable of handling the variabilities in workload demand; this helps reduce the underutilization of over-dimensioned fixed nodes for processing eventual peak demands.

An algorithm, called UAV Fog Node Location (UFL), is introduced in this paper to determine the best combination of fixed nodes and UAVs in an infrastructure. The UFL algorithm initially finds an exact solution to the fog node location problem considering only fixed nodes, and then attempts to replace underutilized servers in fixed nodes by UAVs which can change their location to cope with processing demand at different locations. Simulations considering diverse demand patterns across a metropolitan area as well as real UAV characteristics such as cost, battery capacity and processing capabilities [10] are 
employed to address the question: are UAVs worth adopting to replace fixed nodes in a fog infrastructure? The wireless communication channel is considered ideal, so that results are actually a bound on the value of UAVs used as processing nodes. Results show that the current cost of UAVs is a limiting factor to their usage as fog nodes. In the future, however, and assuming that costs continue to decrease, UAVs could provide interesting solutions for optimally infrastructure dimensioning. Results show that when UAV costs are the same as those of fixed nodes, UAV deployment is indeed advantageous. In this case, the coverage of several locations using UAVs reduces CAPEX and provides more flexibility in coping with unexpected and timely increases in processing demands.

The remainder of this paper is structured as follows. First, previous work is reviewed. The problem in this paper is presented and the experimental setting and numerical results are then discussed. Finally, conclusions are drawn and future work suggested.

\section{UAVS AS NETwORK EDGE ELEMENTS}

UAVs can be used to extend the communication network infrastructure as processing nodes at the edge of the network. One common question in these usages is where to position UAVs to achieve some target objectives. The facility location problem is a design problem in which a set of candidate locations that can host facilities is given as input, with the solution to the problem being the subset of locations for the deployment of these facilities.

Optimizing the location of edge facilities has been explored in previous work considering objectives, such as the cost of facilities [8], [11] and energy consumption [9]. In a cost-based formulation [8], a mixed-integer linear programming (MILP) model was used to obtain the best fog deployment to serve the largest workload at the lowest cost. Another approach [11] considered cloudlets in the deployment and proposed an MILP model to achieve a trade-off between deployment cost and latency experienced by end users. The results obtained by these studies concluded that infrastructure providers can reduce the deployment cost if some degradation in service can be tolerated [8], [11]. The solution in [9] optimizes the number of users served; the reduction in energy spent by mobile devices accessing the infrastructure is considered to be a secondary objective. Fog nodes are deployed in locations where they bring possible opportunities for maximum offloading with minimal energy. A limitation in these approaches is that they consider only fixed fog nodes, but actually the demands on fog nodes are variable. Consequently, a large number of servers are underutilized most of the time in these nodes. 
UAVs are expected to be part of future 6G networks, serving as base stations (BSs) [4], [5], processing nodes [6], [7], and even as users [12]. The authors of [12] considered UAVs to be users of terrestrial base stations and optimized the trajectory followed by UAVs to minimize the task completion time while offloading workload to the ground BSs. The SkyCore architecture for LTE networks [4] proposes the use of unthetered UAVs as base stations, deployed jointly with ground stations. In the three-layer architecture envisioned in [5], UAVs provide coverage to ground users with resources located in satellites, known as CubeSats, furnishing connectivity for ground users by connecting them to satellites.

The UAVFog architecture considered the use of drones as fog nodes [6]. The authors discussed the integration of UAVs with the cloud in monitoring applications, but they did not discuss the battery limitation of drones. The authors in [7] considered the employment of UAVs equipped with servers as processing nodes at the edge hovering at different areas to provide a processing capacity for mobile users. The study showed that the employment of UAVs significantly increased the number of users served in relation to those served in an infrastructure with only fixed nodes. One major drawback of this solution is the consideration of unlimited flight time. Although it is possible to extend UAV flight time by using solar power or tethered drones, such solutions cannot be generalized, since not all aircraft can carry the solar panels. Moreover, tethered drones impose extremely limited mobility.

This paper aims at investigating open questions in previous work. Previous solutions in [8], [11], and [9] either suggest using underutilized servers or allow degradation in quality of service (QoS). In an attempt to overcome these limitations, we have investigated the employment of mobile nodes to extend the capacity of fixed servers, thus reducing resource underutilization. Previous work on the employment of UAVs in cellular networks [4], [5], [12] did not explore the possibility of UAVs as processing nodes.

Table I summarizes the work reported in this section, classifying each paper according to the problem studied. Our present paper considers the employment of UAVs as processing nodes and their battery capacity. Energy consumption is accounted for both processing and flying constituting a more realistic scenario than those evaluated in previous work.

\section{Fog Node LocAtion PROBLEM}

\section{A. System Model}

We consider a system in which mobile users in a metropolitan area request services at different locations, and the processing demand at these locations is a function of user mobility. Applications such as augmented 
TABLE I: Comparison of related work.

\begin{tabular}{|c|c|c|c|c|c|}
\hline Reference & Problem & Criteria & Solution & UAV & $\begin{array}{l}\text { UAV } \\
\text { Battery }\end{array}$ \\
\hline$[8]$ & Fog node location & Workload acceptance and deployment cost & MILP & No & - \\
\hline [9] & Fog node location & Workload acceptance and energy consumption & MILP and Heuristic & No & - \\
\hline [11] & Cloudlet location & Deployment cost and delay & MILP & No & - \\
\hline [12] & Trajectory optimization & Completion time & Heuristic & Yes & No \\
\hline [4] & UAV base stations & User coverage & Architecture and testbed & Yes & Yes \\
\hline [5] & UAV base stations & User coverage & Architecture & Yes & No \\
\hline [6] & UAV fog nodes & User coverage & Architecture & Yes & No \\
\hline [7] & Mobile server location & Workload acceptance & Heuristic & Yes & No \\
\hline Our paper & Fog node location & Workload acceptance and deployment cost & Heuristic & Yes & Yes \\
\hline
\end{tabular}

reality and traffic navigation have strict latency requirements and cannot be processed in the cloud; and users need to connect to a nearby fog node to offload the processing of these applications. If there is no fog node available, requests are rejected (blocked) since they cannot be migrated to more distant nodes due to latency requirements.

A fog node is a small facility that has processing, storage, and networking capabilities. It can process workload offloaded by end users without the need to send it to the cloud through the Internet, thus considerably reducing the response time of applications. Fog nodes serve users in their coverage area, and nodes can have more than one compute server. However, not all servers of a node are continuously needed since the processing demand varies over time.

UAVs can travel from one node to the other to increase the processing capacity of a destination node. UAVs can land to process the workload instead of just hovering. When landed, UAVs have greater autonomy, since the energy consumption of a UAV is much lower than when hovering [10], with energy consumed only by communication and processing.

UAVs can be in one of four different states: turned-off, stand-by, processing, and flying. UAVs are initially turned off, with their battery fully charged. When a UAV is turned off, it does not consume energy. When its service is needed, a UAV starts its operation and remains on until service is no longer required. In the stand-by state, a UAV is landed but not processing and its energy consumption is fixed; moreover, it can be quickly switched to the processing or flying state. In the processing state, the UAV is also landed, but it consumes energy for processing and data transmission. Finally, in the flying state, the UAV is moving between different locations; flights are allowed only to complement the capacity of a fog node at the destination. The flying state is the one that consumes the largest amount of energy, with a consumption depending on the distance traveled and the speed of traveling, both horizontally and vertically. Other sources of consumption such as environmental factors are not considered in this paper. 


\section{B. Problem Statement}

The fog node location problem consists of deciding on the locations where fog nodes should be deployed. The main input to this problem is the set of potential locations for hosting fog nodes, the workload demand, and the available budget for acquiring fixed and UAV fog nodes. The output is the set of locations selected for the deployment of nodes as well as the number of servers at each node. Additionally, the number of UAVs and their flight plan should be determined. The primary goal is to process the maximum possible amount of workload, and the secondary goal is to reduce the infrastructure cost.

The proposed fog node location problem is a bi-criteria optimization problem and can be modeled as a mixed-integer linear programming formulation. In the formulation, time is discretized and the workload at each location is accounted for each time slot. The capacity and cost of fixed and UAV nodes are given. The energy spent in all states as well as the UAV battery capacity are provided as input to the optimization problem. The output is the workload processed at each location for every time slot, the number of fixed servers at each location, the number of UAVs employed, the state, and the location of all UAVs at every time slot.

Such deployment planning is a network design problem, and, as such, is typically solved off-line. However, solving this problem optimally using existing solvers does not scale to large problem instance, for example considering all possible flight combinations. To circumvent these limitations, a heuristic algorithm is proposed next.

\section{UAV Fog Node Location Algorithm}

We propose a heuristic algorithm called the UAV Fog Node Location (UFL) algorithm. Figure 1a shows its flowchart. The algorithm starts by solving the formulation with only fixed nodes, and the pre-defined budget limiting the number of servers and nodes that can be deployed. Based on the solution obtained, the algorithm identifies servers that can potentially be replaced by UAVs; these servers are typically underutilized and deployed only to deal with peak demands. The UFL algorithm then attempts to use UAVs to cover several locations at different times to reduce the deployment cost. The algorithm considers UAVs and fixed servers to have the same cost. After the UFL algorithm is executed, solutions can be produced considering different cost ratios.

The first step of the algorithm considers only fixed nodes with the result obtained using an optimization solver (Step 1). The next step is the identification of servers to be replaced (Step 2). For each fog node, 


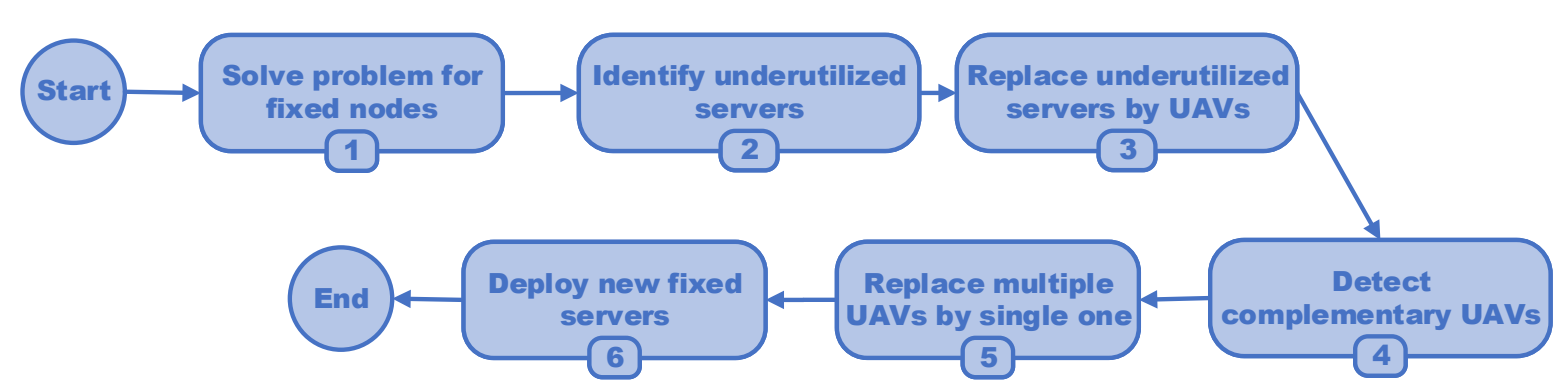

(a) Algorithm flowchart.

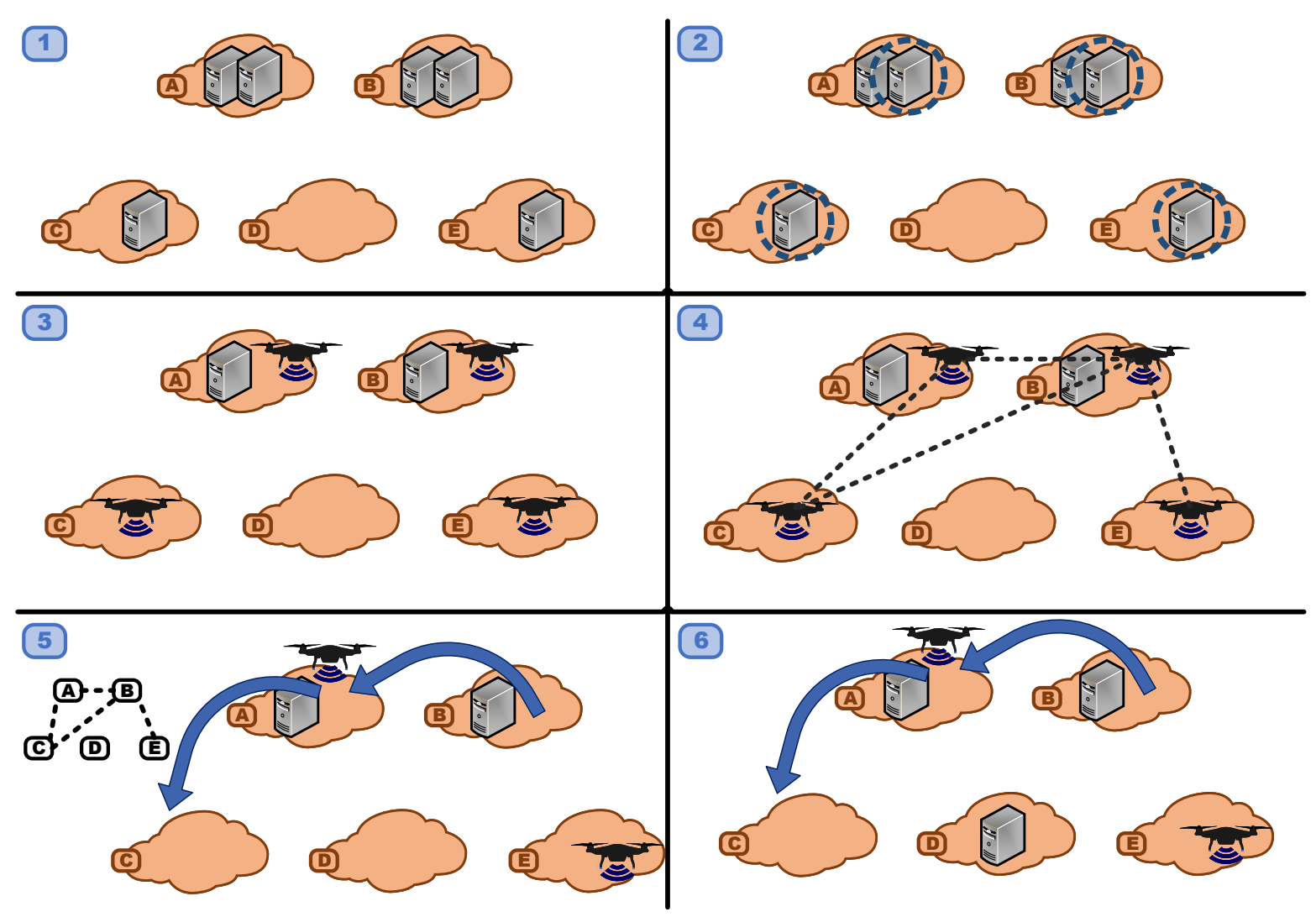

(b) Example of execution.

Fig. 1: UFL algorithm.

the algorithm identifies if a server can be replaced by a UAV, a situation which arises if a server is not processing requests for all time intervals, the UAV processing capacity is greater than the offered workload, and the energy that will be consumed by the UAV is less than its available battery capacity. The energy needed is the sum of the energy spent in processing and that in stand-by in periods associated with the potential replacement. The identified servers are then replaced by UAVs (Step 3).

To reduce the infrastructure cost (secondary objective), all pairs of UAVs are considered to be replaced by a single UAV (Step 4). Two UAVs can be replaced by a single one if three conditions are fulfilled. 
First, the two UAVs must be in processing state in different time periods. Second, the time for travelling between the two locations is less than the time elapsed between the end of the processing at the node for which the UAV departs and the beginning of processing at the destination node. Third, the UAV battery should be sufficient to support full operation, including the flight between the fixed fog nodes.

The algorithm evaluates a potential reduction in the number of UAVs (Step 5). Such an evaluation is carried out by considering a graph in which each UAV is a vertex and each potential pair of locations for replacement is an edge of the graph. Then, the algorithm finds maximal cliques, which determines the minimum number of UAVs to be deployed. If the solution still leaves a backlog of unprocessed workload and the number of UAVs has been reduced in the last step, the unused budget can be employed to further reduce the unserved workload (Step 6).

Figure $1 \mathrm{~b}$ exemplifies the steps involved in planning fog nodes in five locations. The budget comprises six servers, and the solution obtained in Step 1 indicates fog nodes in locations A and B with two servers each due to their larger processing demand; other locations have a low processing demand, with only one server in locations $\mathrm{C}$ and E. Four fixed servers are identified as being underused in Step 2, and are then replaced by UAVs (Step 3). In Step 4, the algorithm detects the pairs of servers that have complementary processing demands in time, and that the battery of a single UAV being adequate to support the operation in both locations; the dashed lines indicate these pairs. Step 5 shows the graph of UAVs, which contains two maximal cliques. Locations A, B, and C can be served by a single UAV. Since one UAV has replaced three fixed servers, an extra server can be deployed in location D, thus increasing the overall workload served (Step 6). The final solution has three fixed servers and two UAVs, thus employing fewer devices than the initial solution.

The UFL algorithm, as presented here, considers that any fixed server can be replaced by a UAV server. If the price of a UAV is greater than that of a fixed server, the solution obtained may not always be worth adopting. As a consequence, alternative solutions should be obtained which consider different ratios between the cost of a UAV node and that of a fixed node. Such an analysis facilitates long term planning for the evaluation of the infrastructure, thus helping avoid unnecessary expenses in the deployment of the original infrastructure.

\section{Evaluation OF THE UFL ALGORITHM}

To answer the question of whether UAVs are worth adopting for replacing fixed fog nodes, extensive simulations of the UFL algorithm involving realistic scenarios were carried out. 
TABLE II: Parameters adopted in the UAV simulations. $H$ refers to the vertical distance travelled, either upwards or downwards.

\begin{tabular}{ll}
\hline Operation & Energy model \\
\hline Fly horizontally & $245.2815 \mathrm{~W}$ \\
Fly vertically up & $\left(-16.9396 H^{2}+216.6944 H-157.9473\right) \mathrm{J}$ \\
Fly vertically down & $\left(4.6817 H^{2}-11.9708 H+135.3118\right) \mathrm{J}$ \\
Stand-by state & $8.2637 \mathrm{~W}$ \\
Processing state & $15.7637 \mathrm{~W}$ \\
\hline & \multicolumn{1}{c}{ Speed } \\
\hline Horizontal & $10 \mathrm{~m} / \mathrm{s}$ \\
Vertical up/down & $1 \mathrm{~m} / \mathrm{s}$ \\
\hline & Variable parameters \\
\hline Battery capacity & $4500 \mathrm{mAh} / 14.8 \mathrm{~V}$ \\
& $34200 \mathrm{mAh} / 22.8 \mathrm{~V}$ \\
Processing capacity & $50 \%$ and $100 \%$ of a fixed server \\
UAV price & 1 and 4 times the price of a fixed server
\end{tabular}

\section{A. Scenario and data description}

The parameter values defining the scenarios in the simulations are summarized in Table II. The UAVs considered are multi rotor drones which have the capacity to land in limited spaces. The characteristics of the simulated UAVs are based on real drones described in previous work [4], [10]. In [10], the authors measured the energy consumption of an Intel AeroReady to Fly Drone and derived models to estimate the energy consumption for diverse operations. These models are used to simulate the energy consumption in vertical and horizontal flights, as well as the energy consumed in the stand-by state. The energy for the processing state is calculated as the energy in stand-by plus the energy spent by a Jetson TX2, a typical on-board computer for drones. The speed of the drone was based on the Intel AeroReady to Fly Drone.

Since the UAV used in [10] does not have a powerful battery, the present evaluation also considers different battery models [4]. Moreover, two other parameters were varied as a function of the fixed servers: the UAV processing capacity and UAV price.

The locations and the workload demands were based on real data made publicity available by Telecom Italia [13]. This data set was collected using Call Detail Records (CDR) of mobile users between November and December, 2013 in the metropolitan region of Milan, Italy. This paper uses the CDR with information about Internet accesses to model the workload, since these records include various applications. The geographical area of the data set was represented as a 100x100 grid, with each cell having its own CDR information. These CDRs are aggregated in intervals of a 10-minute duration.

The cells identified in the data set [13] cannot be used directly as the set of locations. Actually, users in the data set can request services from a base station (BS) that can be located in a different cell. Since the location of base stations was not included in the data set [13], data from the OpenCellId project [14] 
were used to obtain the locations. The OpenCellId is a public database with location information of base stations worldwide collected by mobile users. The location of the base stations was obtained by filtering the existing base stations for the same period available in the Milan data set [13]. The workload of each cell was mapped to the closest base station, as in [15]. In the case of multiple base stations in a cell, the workload is equally balanced among the BSs.

After processing the two databases, the set of location is formed by the base stations from the OpenCellId project, with the workload for each location taken from the Milan data set [13]. The geolocation of base stations is used to calculate the distance between locations, both horizontal and vertical. These data were used to calculate the time UAV would need to travel and, consequently, the energy required for these trips. The capacity of the server was 500 processing units per time interval, and the duration of each interval 10 minutes, which results in 144 intervals in 24 hours. Moreover, 1150 locations were considered.

\section{B. Numerical Evaluation}

The UFL algorithm was coded in Python, and its first step (bi-criteria formulation for the deployment of fixed nodes only) was solved using the Gurobi Optimizer solver. The results produced by the UFL algorithm were compared to those obtained by the solver. Two metrics were evaluated, related to the two objectives of the problem: the acceptance ratio of workload and the number of devices deployed (servers and UAVs). The first metric is the ratio between the workload served and the total workload requested by the end users. The second metric is the number of servers used for the solution using only fixed nodes, and the number of servers and UAVs employed computed by the UFL algorithm. Sixty executions were carried out to derive each value with a $95 \%$ confidence interval. The number of available servers for deployment $(N)$ was varied from 1 to 2048. $U A V^{C}$ denotes the ratio between the cost of a UAV and the cost of a fixed server. Similarly, $U A V^{P}$ is the ratio between the processing capacity of a UAV server and that of a fixed server.

The acceptance ratio using UAVs with the most powerful battery and the same capacity as a fixed server is shown in Figure 2. The acceptance ratio increases until $N=1280$, when servers are sufficient to deal with all the demand. The demand served depends predominantly on the fixed infrastructure capacity due to the limited autonomy of UAVs to stay powered for long periods. Nevertheless, improvements were noticed when UAVs and fixed servers have the same cost and $N \geq 128$ since UAVs could be widely deployed. In these cases, UAVs improved the acceptance of workload and, in some cases $(N \geq 1536)$, 


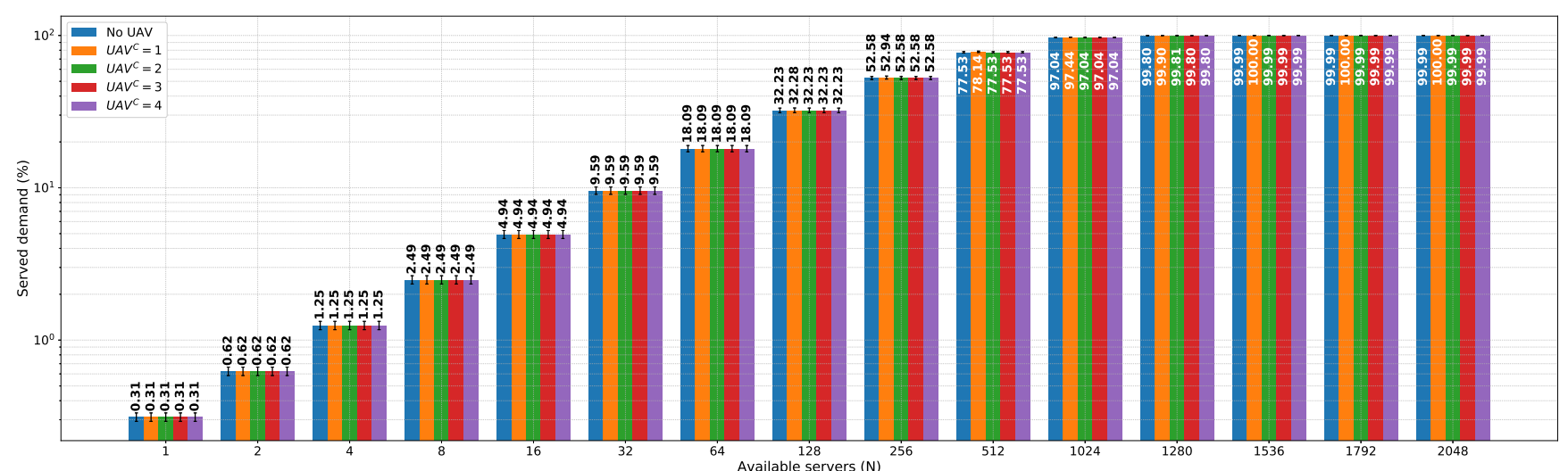

Fig. 2: Acceptance ratio for the $34200 \mathrm{mAh} / 22.8 \mathrm{~V}$ battery and $U A V^{P}=1$.

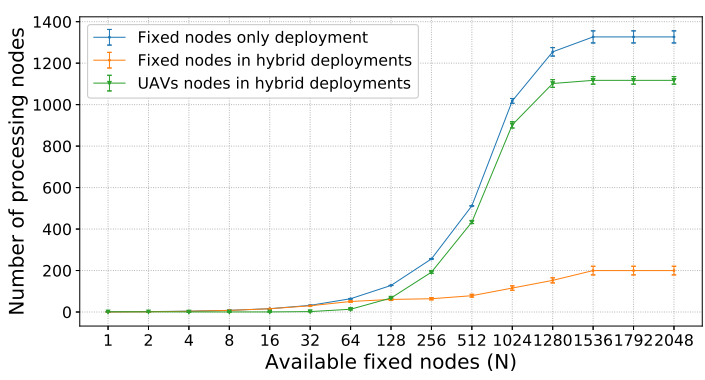

(a) $34200 \mathrm{mAh} / 22.8 \mathrm{~V}$ battery, $U A V^{C}=1$ and $U A V^{P}=1$.

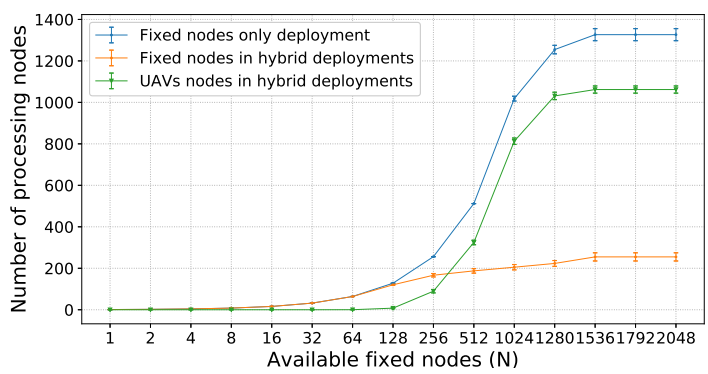

(c) $34200 \mathrm{mAh} / 22.8 \mathrm{~V}$ battery, $U A V^{C}=1$ and $U A V^{P}=0.50$.

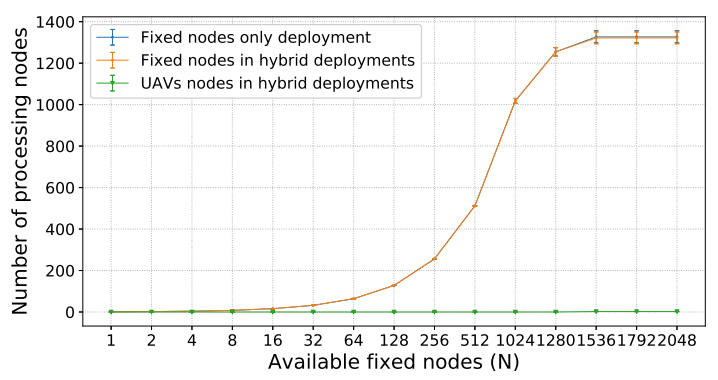

(b) $34200 \mathrm{mAh} / 22.8 \mathrm{~V}$ battery, $U A V^{C}=4$ and $U A V^{P}=1$.

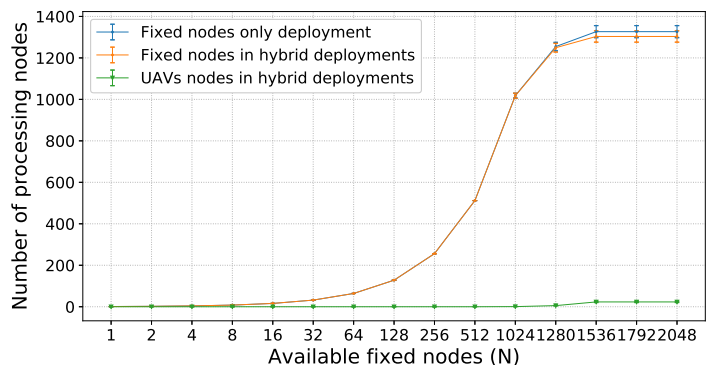

(d) $4500 \mathrm{mAh} / 14.8 \mathrm{~V}$ battery, $U A V^{C}=1$ and $U A V^{P}=1$.

Fig. 3: Number of servers and UAVs used for various scenarios.

provided $100 \%$ acceptance of workload. Higher costs of UAVs limited their number considerably and, as a consequence, the workload acceptance did not change in relation to deployment with only fixed nodes.

The results described below show the impact of the cost of UAVs, UAV processing capacity, and their autonomy on the deployment. Variations in these parameters do not make significant changes in the workload acceptance when compared to those already shown (Figure 2). This is due to the fact that the workload acceptance is predominantly optimized by the fixed servers, with only slight improvements by the effect of using UAVs. In line with these results, only number of UAVs and servers are considered in the following experiments. 
The number of employed devices is shown in Figure 3 for an optimal deployment with only fixed nodes as well as for hybrid deployments (fixed servers and UAVs) obtained by the use of the UFL algorithm. Figure 3 a shows the results for the greatest battery capacity, with UAV cost and processing capacity equal to those of fixed servers. For $N<128$, almost all fixed servers in fog nodes were heavily used for long periods of time so that replacing servers with UAVs is not possible. When a larger number of devices is available for deployment, a large number of fixed servers is replaced by UAVs, which shows that, despite the large number of locations (1150), only about 200 fixed servers could not be replaced by aerial servers, i.e. an infrastructure with only $20 \%$ of the locations being fixed nodes and UAVs serving the remaining $80 \%$ of the locations.

Nowadays, UAVs cost is three to four times the cost of a traditional fixed server, and, under these circumstances, the employment of several UAVs is not advantageous. Figure $3 \mathrm{~b}$ shows the results for UAVs four times more expensive than a fixed server. For $N \leq 1280$, the average number of employed UAVs is very close to zero. This low number of flying servers is due to the fact that using the same UAV to serve two different locations is not always possible because of the required flight time between the locations, which led to quickly drain of the UAV battery. As shown, the UAV price is decisive to be considered in large deployments.

The results considering UAV servers having $50 \%$ of the capacity of a fixed server are shown in Figure 3c. The greater the capacity, the larger the number of servers replaced by UAVs, since UAVs with a limited processing capacity cannot always deal with the peak demands supported by a fixed server. The increase in the processing capacity and the increase in the number of UAVs is not linear: increasing the UAV processing capacity from $50 \%$ to $100 \%$ leads to an increase in less than $10 \%$ in the number of UAVs for $N>1280$. This is explained by the pattern of the frequency of peak demands. Servers replaced by UAVs are seldom used, and, therefore, they deal with rather sporadic demands. Given these low demands, UAV with powerful computers are not required.

The final analysis concerns the battery capacity. Figure $3 \mathrm{~d}$ shows the results for the UAVs with the smallest battery capacity. This type of battery has a very limited autonomy, thus it has little use in such an infrastructure. Only when all the demand was met $(N \geq 1536)$ can approximately 25 UAVs replace fixed servers. The problem is the autonomy of the batteries, which prevents the replacement of a single underloaded server with a UAV. To further increase the number of UAVs, the battery life would have to be sufficient to maintain the UAVs turned on for several hours, which is not a realistic assumption for 
battery-constrained UAVs. Technologies for charging batteries without interrupting the operation can help to extend UAVs operation in fog infrastructures.

The present findings have revealed advantages and disadvantages in relation to the adoption of such hybrid infrastructures with both fixed and UAV nodes. One advantage is that hybrid infrastructures can simplify the deployment of fixed nodes where processing demands are low, thus reducing costs for deploying and maintaining nodes with underused servers continuously turned on. However, as long as UAV prices are higher than those of traditional servers, their use will remain limited. In case of price reduction, UAVs in fog infrastructures may become much more wide-spread.

\section{CONCLUSiOnS}

This paper has investigated the employment of unmanned aerial vehicles as fog nodes by solving a fog node location problem. By considering UAVs in this early stage, it is possible to plan the best deployment and avoid placing fixed servers in locations with low demand. This paper has described the UFL algorithm which first solves the problem optimally by considering only fixed servers, and then tries to replace underutilized servers by UAVs, which can potentially serve more than one location. Results were obtained by varying different UAVs types and using a publicly available dataset. The UFL algorithm can be used for long-term planning of large fog infrastructures. Results show that a significant portion of the infrastructure could be replaced by UAVs depending on their price evolution. An additional benefit of using UAVs is the energy saved compared to an infrastructure with only fixed servers constantly powered all the time. Our investigation has revealed that such a deployment depends on the prices of UAVs being close to that of traditional servers. Currently, UAVs cost three to four times more than traditional servers, but prices are expected to decrease in the future as a function of mass production and wide use of unmanned aircrafts.

The findings in this paper suggest opportunities for future investigation. Similar solutions can be evaluated for different scales of infrastructure, from small neighborhoods to wider areas. By using fixed wing drones, for example, long hours of flight can be achieved, yet this would require logistics to allow landings and takeoffs. Second, the solution presented in this paper could be adapted to a scenario where the infrastructure already exists and the UAVs are simply added to serve unforeseen and timely processing demands. 


\section{REFERENCES}

[1] N. L. S. da Fonseca and R. Boutaba, Cloud Services, Networking, and Management. John Wiley \& Sons, Ltd, 2015.

[2] “OpenFog Reference Architecture,” Online, 2017, available at https://www.openfogconsortium.org/ra/ [Accessed: 24/05/2017].

[3] X. Masip-Bruin et al., "Foggy clouds and cloudy fogs: a real need for coordinated management of fog-to-cloud computing systems," IEEE Trans. Wireless Commun., vol. 23, no. 5, pp. 120-128, 2016.

[4] M. Moradi et al., "Skycore: Moving core to the edge for untethered and reliable uav-based lte networks," in ACM MobiCom 2018. New York, NY, USA: Association for Computing Machinery, 2018, p. 35-49.

[5] R. Bassoli et al., "Cubesat-based 5g cloud radio access networks: A novel paradigm for on-demand anytime/anywhere connectivity," IEEE Veh. Technol. Mag., vol. 15, no. 2, pp. 39-47, 2020.

[6] N. Mohamed et al., "Uavfog: A uav-based fog computing for internet of things," in IEEE SmartWorld/SCALCOM/UIC/ATC/CBDCom/IOP/SCI 2017, 2017, pp. 1-8.

[7] J. Wang, K. Liu, and J. Pan, “Online uav-mounted edge server dispatching for mobile-to-mobile edge computing,” IEEE Internet Things J., vol. 7, no. 2, pp. 1375-1386, Feb 2020.

[8] R. A. C. da Silva and N. L. S. da Fonseca, "On the location of fog nodes in fog-cloud infrastructures," Sensors, vol. 19, no. 11, 2019.

[9] — - "Location of fog nodes for reduction of energy consumption of end-user devices," IEEE Trans. Green Commun. Netw., 2020.

[10] H. V. Abeywickrama et al., "Empirical power consumption model for uavs," in IEEE VTC-Fall 2018, 2018, pp. 1-5.

[11] Q. Fan and N. Ansari, "Cost aware cloudlet placement for big data processing at the edge," in IEEE ICC 2017, May 2017, pp. 1-6.

[12] X. Cao, J. Xu, and R. Zhang, "Mobile edge computing for cellular-connected uav: Computation offloading and trajectory optimization," in IEEE SPAWC 2018, 2018, pp. 1-5.

[13] G. Barlacchi et al., "A multi-source dataset of urban life in the city of milan and the province of trentino," Scientific Data, vol. 2, Oct 2015

[14] Opencellid. Accessed on 2021.01.12. [Online]. Available: http://www.opencellid.org

[15] L. Chen et al., "Complementary base station clustering for cost-effective and energy-efficient cloud-ran," in IEEE SmartWorld/SCALCOM/UIC/ATC/CBDCom/IOP/SCI 2017, Aug 2017, pp. 1-7. 